\title{
The impact of horizontal gene transfer in shaping operons and protein interaction networks - direct evidence of preferential attachment
} Wagied Davids ${ }^{1}$ and Zhaolei Zhang*1,2

Address: ${ }^{1}$ Banting \& Best Department of Medical Research (BBDMR), Donnelly Centre for Cellular \& Biomolecular Research (CCBR), University of Toronto, 160 College Street, Toronto, ON M5S 3E1, Canada and 2Department of Molecular Genetics, University of Toronto, Toronto, ON M5S 3E1, Canada

Email: Wagied Davids - wagied.davids@utoronto.ca; Zhaolei Zhang* - zhaolei.zhang@utoronto.ca

* Corresponding author

Published: 24 January 2008

BMC Evolutionary Biology 2008, 8:23 doi:10.1/86/147|-2/48-8-23

This article is available from: http://www.biomedcentral.com/I47I-2I 48/8/23

(c) 2008 Davids and Zhang; licensee BioMed Central Ltd.

This is an Open Access article distributed under the terms of the Creative Commons Attribution License (http://creativecommons.org/licenses/by/2.0), which permits unrestricted use, distribution, and reproduction in any medium, provided the original work is properly cited.
Received: 19 August 2007

Accepted: 24 January 2008

\begin{abstract}
Background: Despite the prevalence of horizontal gene transfer (HGT) in bacteria, to this date there were few studies on HGT in the context of gene expression, operons and protein-protein interactions. Using the recently available data set on the $E$. coli protein-protein interaction network, we sought to explore the impact of HGT on genome structure and protein networks.

Results: We classified the $E$. coli genes into three categories based on their evolutionary conservation: a set of 2158 Core genes that are shared by all E. coli strains, a set of 1044 Non-core genes that are strain-specific, and a set of 1053 genes that were putatively acquired by horizontal transfer. We observed a clear correlation between gene expressivity (measured by Codon Adaptation Index), evolutionary rates, and node connectivity between these categories of genes. Specifically, we found the Core genes are the most highly expressed and the most slowly evolving, while the HGT genes are expressed at the lowest level and evolve at the highest rate. Core genes are the most likely and HGT genes are the least likely to be member of the operons. In addition, we found the Core genes on average are more highly connected than Non-core and HGT genes in the protein interaction network, however the HGT genes displayed a significantly higher mean node degree than the Core and Non-core genes in the defence COG functional category. Interestingly, HGT genes are more likely to be connected to Core genes than expected by chance, which suggest a model of differential attachment in the expansion of cellular networks.
\end{abstract}

Conclusion: Results from our analysis shed light on the mode and mechanism of the integration of horizontally transferred genes into operons and protein interaction networks.

\section{Background}

It is generally accepted that horizontal gene transfer (HGT) is an important process in bacterial genome evolution, which provides both novel metabolic capabilities, and catalyzing the diversification of bacterial lineages
$[1,2]$. Although, the extent of the evolutionary impact of HGT is still under debate [3], it is generally accepted that roughly $10-40 \%$ of the protein-coding genes are likely to have been introduced by HGT into the E. coli K12 genome 
[4] since the species divergence from the Salmonella lineage approximately 100 million years ago [5].

Currently, no plausible mechanisms have been proposed for the incorporation of HGT genes into their recipient genomes. We envisage that successful incorporation of a horizontally transferred gene needs not only its successful transcription and translation, but also its integration into the existing functional cellular network. We foresee a number of barriers that potentially exist against the incorporation and expression of horizontally transferred genes in a new recipient genome.

The first step of integration for horizontally transferred genes is its incorporation into the host transcription machinery. Bacterial genes are often organized into groups called operons, which enable a simple and unified mechanism of gene regulation in bacteria. Integrating into operons may be regarded as beneficial for the foreign invading genes, since they gain the opportunity not only to be co-regulated and but also co-expressed with resident genes. Secondly, HGT genes may need to optimize their codon usage to be compatible to the host in order to be efficiently transcribed and translated. Thirdly, the protein product has to be integrated into the functional cellular network in order to gain interaction partners and contribute fitness benefits to the organism. Failure to achieve any of the above steps may result in eventual degradation and pseudogenization.

Considering the prevalence of horizontal gene transfer during bacterial genome evolution, the importance of studies exploring their mode of evolution, expression and impact on genomic organization and protein-interactions would thus further our understanding of horizontal gene transfer. With the emergence of high-throughput functional genomics and proteomics data, we are offered a unique opportunity of answering these questions. Thus our specific aims in this paper were to address the following questions:

\section{(i). Evolutionary Rates and Gene Expression characteristics of Core, Non-core and HGT genes}

Bacterial genomes are known to be dynamic, consisting of genes with different evolutionary histories. Some genes are evolutionarily conserved while others can be gained and lost in a lineage-specific fashion, and by horizontal gene transfer events. Prior studies on yeast and vertebrates have suggested that genes that are the most evolutionary conserved and most highly expressed evolve at the slowest rate $[6,7]$. Therefore to investigate the effect of selection on these various gene categories, we classified $E$. coli genes according to their evolutionary conservation into Core, Non-core and HGT genes (see Methods). In this regard, we hypothesize that the cumulative effect of selection acting on these different gene categories would leave footprints in their sequence and gene expression characteristics.

\section{(ii). The contribution of HGT to operon formation}

It is known that horizontally transferred genes can be inserted into existing operons and thus contribute to the dynamic nature of the gene order and membership of these operons [8-15]. Although a few studies have investigated the evolutionary stability and the conservation of gene order of operons $[16,17]$, the relative contribution of HGT on the evolutionary composition of operons remains unclear. In this regard, we aimed to explore the prevalence of HGT genes in operons by cataloguing the presence of operons consisting of Core, Non-core and HGT genes.

\section{(iii). The impact of HGT on protein-protein interactions and networks}

Another area that has been missing in the study of HGT events is the aspect of protein-protein interactions and cellular networks. A few studies have concentrated on the impact of horizontal gene transfer on metabolic networks $[18,19]$. Unfortunately very little is known about the effect of horizontal gene transfer on the global protein interaction networks in this aspect, mostly due to the lack of cellular interaction data in bacteria until recently.

It has been suggested that the scale-free properties of biological networks may in part be due to a model of preferential attachment by means of gene duplication, whereby new nodes preferentially attach to existing highly connected nodes. In networks that have evolved via preferential attachment, older nodes should have a higher average connectivity than younger nodes [20]. In this regard, horizontal gene transfer can be considered as an additional biological mechanism to the existing model of preferential attachment. Although distinctly different, a model of network growth and expansion that involves gene duplication results in a duplicate protein copy with exact same or similar function, whereas a mechanism involving HGT may represent novel functions. In this regard, proteins encoded by HGT genes can be seen as competing with resident genes in establishing and gaining protein interactions.

We investigated both operons and protein interactions as a means of detecting successful incorporation of putative horizontally transferred genes in the E. coli genome. We explored the possibility that successful HGT genes would require integration at the level of operons to be expressed and integration at the network level to establish fitness benefits to the organism. We found horizontally transferred genes exhibit lower gene expressivity and evolve at faster evolutionary rates than evolutionarily conserved core genes. In addition, although proteins encoded by 
horizontally transferred genes have lower network connectivity, they preferentially attach to resident Core proteins rather than Non-core proteins within the protein interaction network. We conclude that a small proportion of the low connectivity proteins may have arisen from HGT events.

\section{Methods \\ Data}

Genome sequences available for the various E. coli strains were downloaded from the NCBI (Escherichia coli K12 MG1655 - NC_000913; Escherichia coli O157H7 NC_002695; Escherichia coli O157H7_EDL933 NC_002655; Escherichia coli CFT073 - NC_004431; Escherichia coli UTI89 - NC_007946).

\section{Deriving a set of HGT genes in E. coli}

Our primary data set consisted of horizontal gene transfer events that were identified using a combination of the gene phylogeny and the pattern of gene presence and gene absence [15]. This approach is similar to gene presence/ absence analyses $[21,22]$.

For detection of horizontal gene transfer events, a total of 326 complete bacterial genome sequences divided into 8 bacterial clades were downloaded from MicrobesOnline database [23]. Using each protein sequence contained within the E. coli K12 genome as query, BLASTP sequence similarity searches are conducted against all 326 bacterial proteomes. Subsequent BLAST sequence hits are further categorized into "BestN" hit categories with the Best0 category referring to the E. coli $\mathrm{K} 12$ gene itself. Each gene is assigned to a relative age category (i.e. clade) based on the BLASTP hit with the highest score. The method classifies each gene within the E. coli $\mathrm{K} 12$ genome as belonging to either (i) a set of horizontally transferred gene set (named HGT), (ii) a native gene set restricted to the $E$. coli lineage (named Native) or (iii) a gene set with no known sequence homologs (named ORFan). Thus the BLASTP scores gradually decrease in groups with increasing phylogenetic distance from E. coli K12.

Multiple sequence alignments based on protein sequences are then constructed using the MUSCLE sequence alignment software [24]. Fast neighbour-joining trees [25] are then subsequently constructed for each protein sequence alignment. Genes that lack "close" homologs in consecutive groups of related bacteria are then confirmed using a quartet test available within the software package TREE-PUZZLE [26]. To infer a horizontal gene transfer event; gene trees are compared with the MicrobesOnline specie tree (see above). If a strongly supported clade in the gene tree was present in disparate genomes, so that three or more deletion events would be required to explain the distribution of the subfamily on the species tree, then an HGT event was assigned.

In addition, we have included a comparison of horizontally transferred genes obtained by various HGT detection methods which comprised three surrogate (non-tree based) methods namely, HGT-DB [27], the method published by Mrazek and Karlin [28] and a support vector machine-based method (HGT_SVM) developed by Tsirigos and Rigoutsos [29] versus our data derived from a combined phylogenetic and gene presence/absence based method [15], both in terms of overall counts but also in terms of their distribution of Cluster of Orthologous (COG) functional categories (see Additional File 1).

On the overall, the method developed by Price et al. predicts more HGT genes in E. coli $\mathrm{K} 12$ than the three surrogate methods. It is known that base compositional differences between resident and invading genes are "ameliorated" over a few million years [30]. Surrogate methods that use a compositional approach may preferentially detect recent horizontal gene transfer events and genes with atypical base compositions [21]. Thus, a crossphylum approach using phylogenetic tree based methods combined with gene phyletic profiles are more likely to detect ancient but also recent horizontal gene transfer events.

Deriving a set of Core and a set of Non-core E. coli genes Our operational definition of a Core set of genes was meant to reflect the evolutionary retention of a set of common genes in all E. coli strains. In this regard, our Non-core set reflect genes that are found in at least one strain but not all strains, and HGT genes correspond to genes which are derived from putative recent horizontal gene transfer events. Thus, this distinction between Core and Non-core genes serves to illustrate the difference between a stable and invariant Core component and a variable Non-core component that is specific to $E$. coli strains. In this regard, the Non-core genes represent genes with a restricted phylogenetic distribution limited to one or more E. coli strains. These genes can be lost or gained in a strain-specific manner. Thus to ensure that there is no overlap between any evolutionary gene categories we have filtered the Core, Non-core and HGT gene sets to ensure a non-overlapping set of each gene category is maintained.

We derived an evolutionary Core set of 2158 E. coli genes based on the criteria of using phylogenetic gene conservation and genomic context (positional gene conservation). Starting with an all-vs-all protein sequence comparison consisting of the five E. coli genomes, we grouped E. coli K12 genes based on their phylogenetic gene conservation profiles within all five strains. To ensure a high quality Core gene set, we extracted and compared the chromo- 
somal locations of all Core genes. It is known that genes which evolve vertically between closely related species can be divided into those that retain homologous chromosomal positions (positional orthologs) and those that do not [31]. In addition, phylogenetic trees were constructed based on selected protein sequences to verify the phylogenetic relationship between the five $E$. coli strains.

Our Non-core gene set was obtained by post-process filtering the BLAST sequence comparison results of E. coli $\mathrm{K} 12$ genes which had BLAST hits in at least one or more E. coli genomes, but not present in all genomes. We also extracted and compared gene chromosomal locations of this gene set and constructed phylogenetic trees for further investigation. Since this gene set showed lower phylogenetic conservation, they were also positionally conserved to a lesser extent.

In addition, results from correspondence analysis of codon usage also revealed a distinction between our Core, Non-core and HGT gene categories (Additional File 2). The Core,Non-core and HGT gene lists can be found in Additional Files.

\section{E. coli operons}

Data pertaining to Escherichia coli operons and transcriptional units were downloaded from RegulonDB version 5.7 [32,33]. RegulonDB is a manually curated database that focuses on transcriptional regulation in $E$. coli with information extracted from literature as well as sequence databases such as GenBank. Its basic structural unit is the operon, which describes the elements and properties of transcriptional regulation. Thus in keeping with this definition, we refer to an operon as a poly-cistronic transcribed unit with its associated regulatory sites, whereas a regulon is defined as a group of operons controlled by a single regulator. As of RegulonDB version 5.7, 4570 E. coli genes have been annotated and organized into 2684 operons.

\section{Analysis of E. coli gene expression}

E. coli K12 MG1655 microarray gene expression data were downloaded from the NCBI GEO microarray database [34]. We selected the GDS2600 data set, which closely approximates growth under normal conditions. This data set contains a time course which monitors the expression of $4405 \mathrm{E}$. coli genes using spotted cDNAs in stationary phase using LB media. Log2-transformed gene expression values were used and we excluded genes with missing data from the analysis. For each gene, mean gene expression values across time points were calculated and used for subsequent analysis.

\section{Protein-Protein interaction networks}

For construction of the E. coli interaction network, we extracted the protein-protein interaction data from a recently published mass spectrometry study [35]. We examined this data set carefully to confirm that it was not biased towards particular pathways or functional categories using the KEGG pathways and COG functional classification databases respectively. The whole analysis was also re-performed using the protein interaction data from Arifuzzaman et al. [36] (Additional Files 3 and 4).

\section{Software}

Detection of orthologs was performed using a reciprocal best-hits approach as implemented in the RSD-algorithm [37]. Multiple sequence alignments were constructed from protein sequences using the ClustalW package [38]. Phylogenetic tree reconstructions were performed using the neighbour-joining method [39]. Evolutionary substitution rates were estimated using the CODEML program available from the PAML package[40]. Network analyses were performed using algorithms implemented in the NetworkX package [41] and visualised using PAJEK [42]. Statistical analyses were performed using the R-programming language environment.

\section{Results and Discussion}

HGT genes evolve faster and have lower expression levels To investigate the selective pressure acting on organizational units, we classified E. coli genes according to their evolutionary conservation into three categories, namely, (i). Core Set: a conserved core set of genes that exist in all E. coli strains. (ii). Non-core Set: genes that are found in at least one strain but not in all strains, and (iii). HGT Set: genes that are derived from putative recent horizontal gene transfer events after the divergence of E. coli and Salmonella. By delineating genes according to their evolutionary conservation, we can more clearly identify the evolutionary forces to which the various evolutionary classes of genes are subjected.

Direct measurements of $E$. coli gene expression were obtained from microarray gene expression experiments (see Methods). In addition, we have also used the codon adaptation index (CAI) as a proxy for gene expression data, which we referred as "gene expressivity" [43].

Figure 1 shows that the Core genes have higher CAI gene expressivity levels (Figure 1A) as well as log2 expression values (Figure 1B) than Non-core and HGT genes (t-test and Wilcox rank test, $\mathrm{p}$-value $<0.001)$. This can be explained by the different evolutionary histories of these three groups of genes. The Core set of genes, being the oldest resident genes in the genome have thus had sufficient time to adapt and optimise their codon usage patterns, explaining the higher levels of gene expressivity; whereas 
(A)

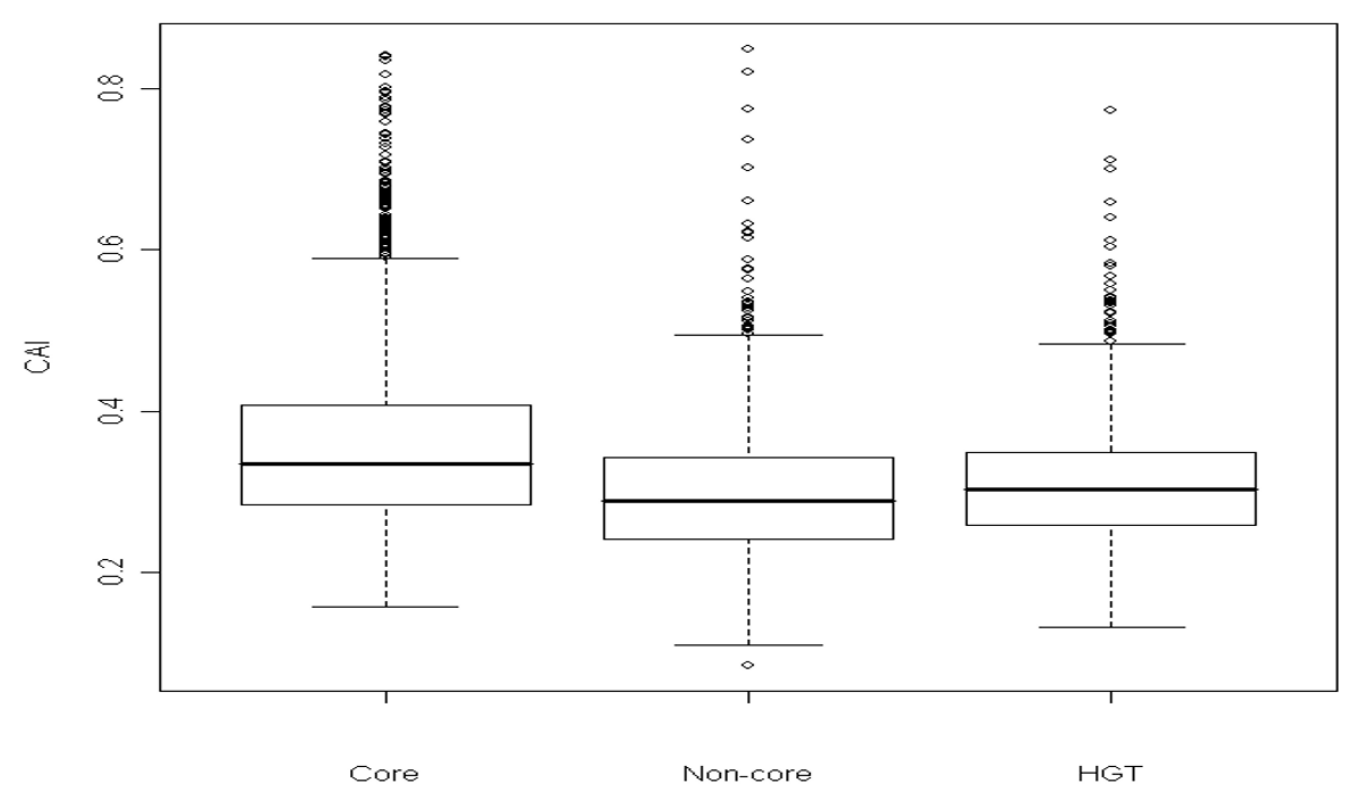

(B)

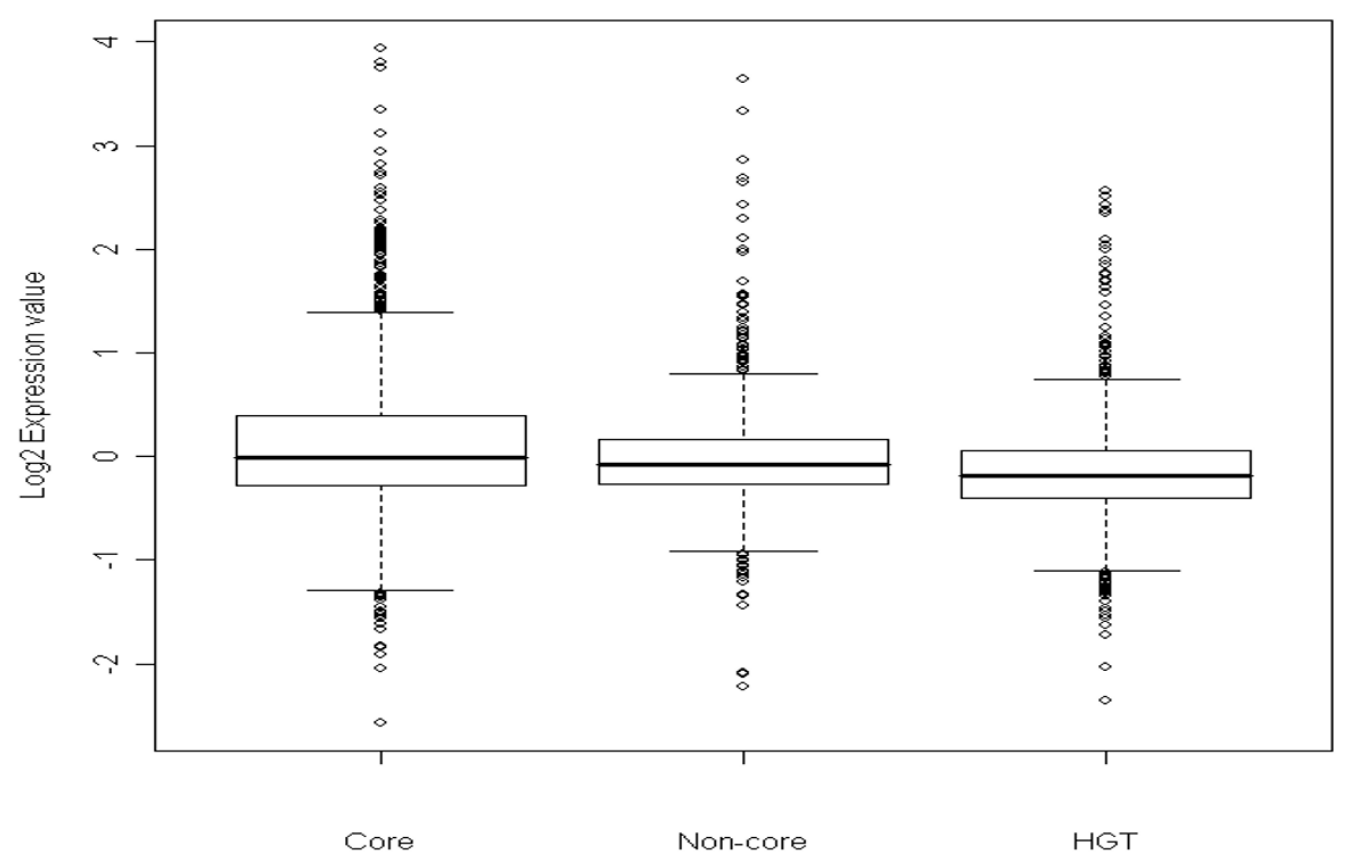

Figure I

Box plot of (A) gene expressivity (CAI) values and (B) log2 gene expression values between Core, Non-core and HGT genes. Core genes display higher expressivity than Non-core and HGT genes (P-value $<0.001$ ). 
the recent horizontally transferred genes may need an adaptation period during which their base composition and codon usage patterns may need to be optimised to their new resident genome.

Figure 2 shows that amongst the three categories of gene sets, the Core set of genes evolve at the lowest substitution rates $(d N / d S)$ and HGT genes evolve at the fastest rates, using E. coli K12 as reference for comparison (WilcoxonMann-Whitney test, p-value $<0.001$ ). The high evolutionary rates observed for HGT genes may be explained by either one of the following two hypotheses: (i) result of reduced negative selection pressure, which enable the invading genes to be purged from the genome, or (ii) result of increased positive selection whereby HGT genes contribute to the phenotypic character of $E$. coli strains [14]. Accordingly, it is thought that the strain-specific Non-core genes and HGT genes may contribute to the pathogenic character separating the enterohemorrhagic and uropathogenic from the benign E. coli K12 strain, therefore these genes are under positive selection pressure.

\section{Genes in Operons and Networks Display Higher Gene Expressivity}

There is increasing evidence to suggest that the chromosomal gene order in organisms is not always random [44]. It is known that proteins of linked genes evolve at comparable rates, and that natural selection may promote the conservation of linkage of co-expressed genes [45]. Accordingly, genes in the same operon occur in close physical proximity and are often known to be co-transcribed as units. In addition, genes encoding subunits of protein complexes also need to be expressed at similar times.

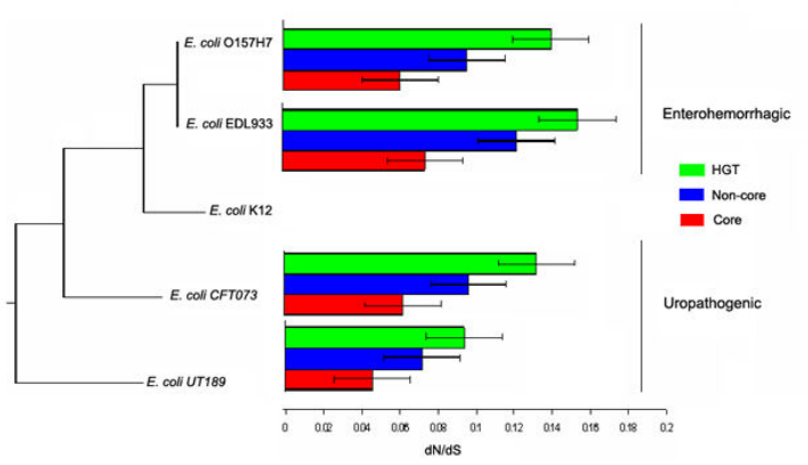

\section{Figure 2}

Distribution of evolutionary rates $(d N / d S)$ for various $E$. coli strains overlaid on a phylogenetic tree using $E$. coli $K / 2$ as reference for genome comparisons. Core genes evolve slower than Non-core and HGT genes (P-value $<0.00 \mathrm{I})$.
To investigate the relative contributions of the various evolutionary gene categories on organizational structures, we surveyed both operons and the protein interaction network for their content of Core, Non-core and HGT genes. The Core set form a predominant portion of operons with $47 \%$ (2129 out of 4506 genes catalogued in RegulonDB version 5.7) of the operons consisting of Core genes, whereas 21\% (948 out of 4506) of Non-core genes and $23 \%$ (1020 out of 4506) HGT genes, respectively, accounted for the remaining gene constituents of operons (Figure 3A). Similarly, proteins encoded by Core genes account for a $67.5 \%$ (852 out of 1262 ) of the protein interaction network as reported by Butland et al [46] whereas Non-core genes and HGT genes account for $14.1 \%$ (178 out of 1262 ) and $18.4 \%$ (232 out of 1262 ) respectively (Figure 3B).

(A)

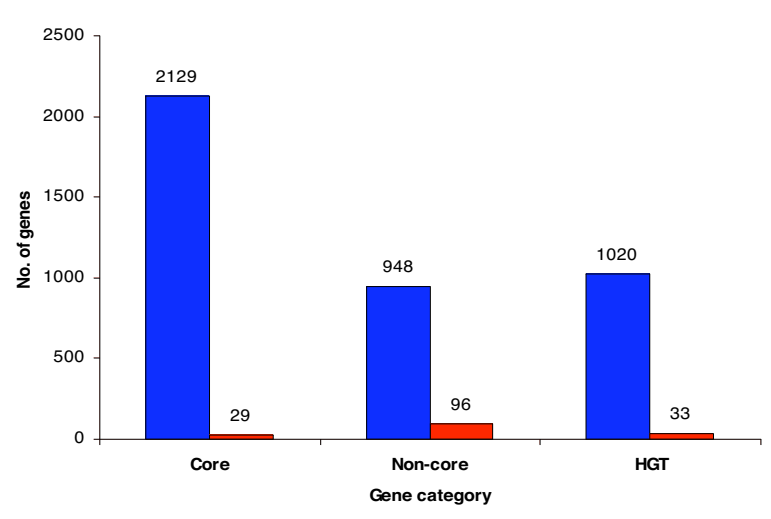

(B)

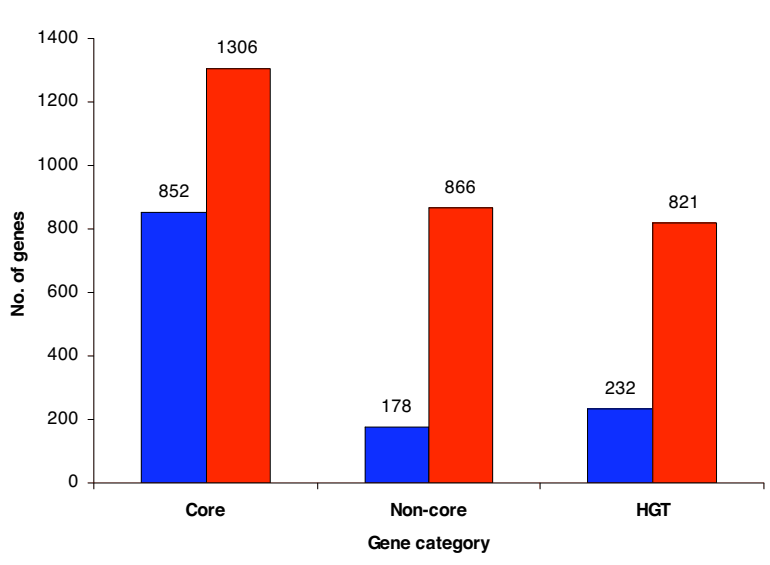

\section{Figure 3}

Number of $E$. coli genes in the genome organizations: (A) operons, (B) protein interaction network (PIN). Genes are classified into three evolutionary categories Core, Non-core and HGT genes. Core genes predominantly occur in both operons and protein interaction networks (P-value $<0.00 \mathrm{I})$. 
The tendency of operons to be enriched in Core genes can be explained by a need to simplify regulation, since genes residing in operons known to be under control of the same promoter (Chi-squared test, $\mathrm{p}$-value $<0.001$ ). This may facilitate horizontal gene transfer by enabling genes to be inherited as a physical and functional cohesive group rather than separate individual genes. In regard to the protein interaction network, it is thought that the Core genes form the ancestral backbones of the protein interaction network to which new functionalities are added via protein nodes and thus strengthens a model by which pathways expand [47].

To understand the impact of higher order organization of genes (i.e. operons) and proteins (i.e. interaction complexes) on properties such as expression or evolution, we

(A)

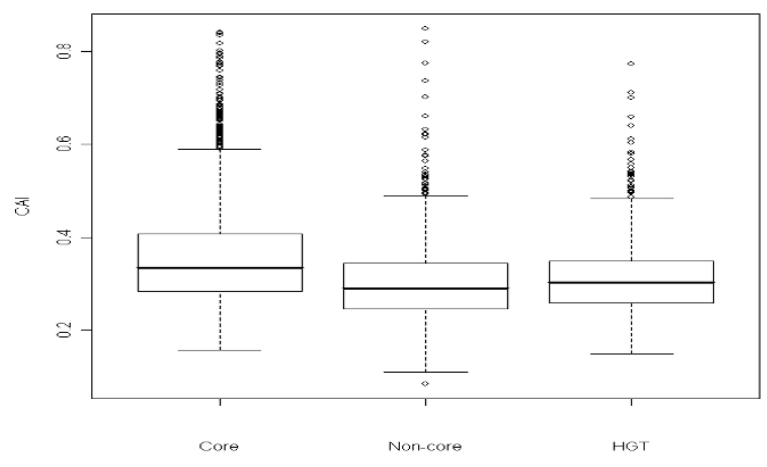

(C)

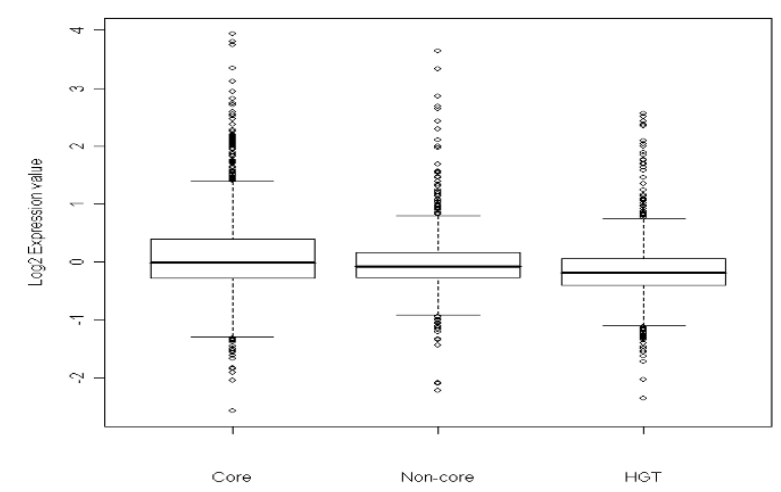

investigated the gene expressivity characteristics and evolutionary substitution rates of the various categories of gene sets. We found that Core genes in organizational clusters (both operons and protein interaction network or PIN) have higher gene expressivity (CAI) values (Figure $4 \mathrm{~A}$ and $4 \mathrm{~B}$ ) and as well as $\log 2$ expression values (Figure 4C and 4D) relative to Non-core and HGT genes (t-test and Wilcox-test for both operons and PIN, p-value $<0.001$ ). For the PIN, this trend was robust against removal of ribosomal proteins.

The overall trend from surveying operons and the protein interaction network indicates that Core genes tend to be found more often in organizational units such as operons and networks. The evolutionary composition may be the reason that highly clustered proteins in the protein inter-
(B)

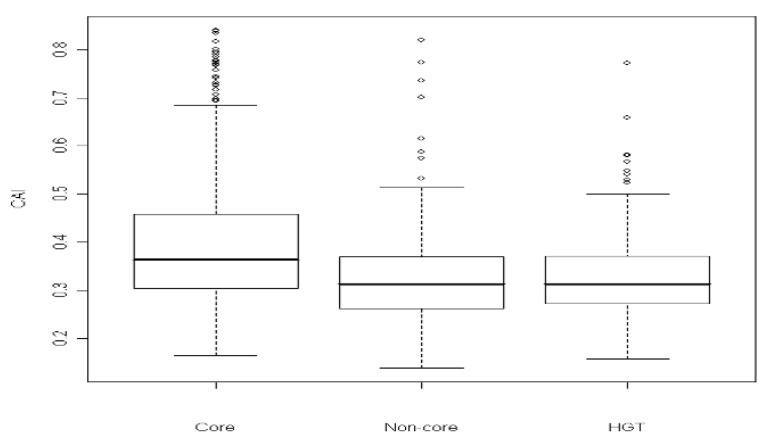

(D)

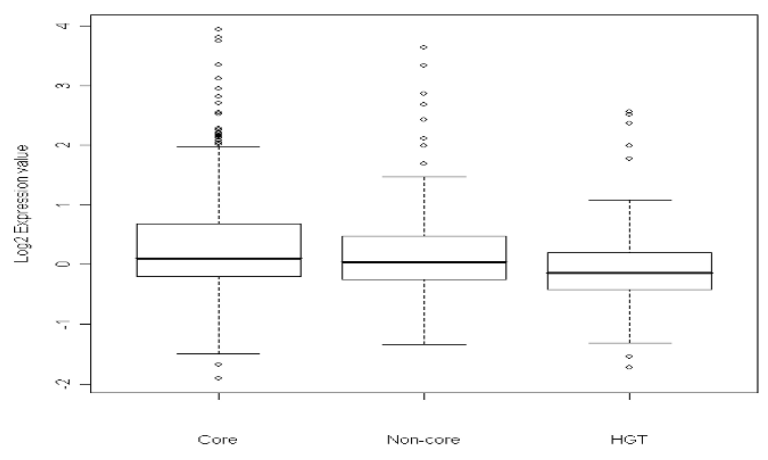

\section{Figure 4}

Gene expressivity (CAI) values and log2 gene expression values between Core, Non-core and HGT genes in different genome organizations. (A) Box plot of CAI values between Core, Non-core and HGT genes in operons; (B) Box plot of gene CAI values between Core, Non-core and HGT genes in protein interaction network (PIN); (C) Box plot of log2 gene expression values between Core, Non-core and HGT genes in operons; (D) Box plot of log2 gene expression values between Core, Non-core and HGT genes in protein interaction network (PIN). 
action network display apparently high gene expressivity and low substitution rates.

Distribution of COG Functional Categories between Core, Non-core and HGT genes within the Operons and Protein Interaction Network

We have analyzed and compared the distribution of the Cluster of Orthologous (COG) functional categories of the Core, Non-core and HGT genes within the E. coli K12 genome, protein interaction network and operons (Figures 5, 6 and 7). The various gene categories differ significantly in their COG distribution in the genome, the protein interaction network and operons (Scheirer-RayHare test, p-value < 0.001, see Additional File 5).

In the overall gene comparison of the E. coli K12 Core, Non-core and HGT gene sets, the Core genes constituted the major evolutionary gene set present in all COG categories (Figure 5). The Non-core gene set in comparison to the HGT gene set was markedly abundant in the two COG categories: O (Posttranslational modification, protein turnover, chaperones) and $\mathrm{T}$ (Signal transduction mechanisms). The HGT gene set was more abundant than the Non-core gene set in the COG categories C (Energy production and conversion), $\mathrm{F}$ (Nucleotide transport and metabolism), G (Carbohydrate transport and metabolism), I (Lipid metabolism), $\mathrm{K}$ (Transcription) and V
(Defense mechanisms). For the operons, the Core genes occur predominately in all COG functional categories, whereas the Non-core genes are over-represented in COG categories S (Function unknown) and U (Intracellular trafficking, Secretion, and vesicular transport) and the HGT genes are over-represented in comparison to the Non-core genes in COG functional categories C, E (Amino acid transport and metabolism), G, H (Coenzyme metabolism), R (General function prediction only) and $\mathrm{V}$ (Defence mechanisms) (Figure 6).

For the protein interaction network, the HGT genes are over-represented in COG functional categories most notably $\mathbf{C}, \mathbf{G}, \mathbf{H}$, and $\mathbf{V}$ (Figure 7). A most notable example in this regard is the COG category $\mathrm{V}$ in which the HGT gene set within the E. coli protein interaction network has a significantly higher mean node degree than the Core and Non-core genes sets. The overall statistical difference in distribution of COG functional categories between the Core, Non-core and HGT gene sets therefore seems to argue against the notion of a Core-versus-Non-core or Core-versus-acquired gene category consisting of Non-core and HGT genes, but rather strengthens the notion of a distinct separate category for Non-core genes.

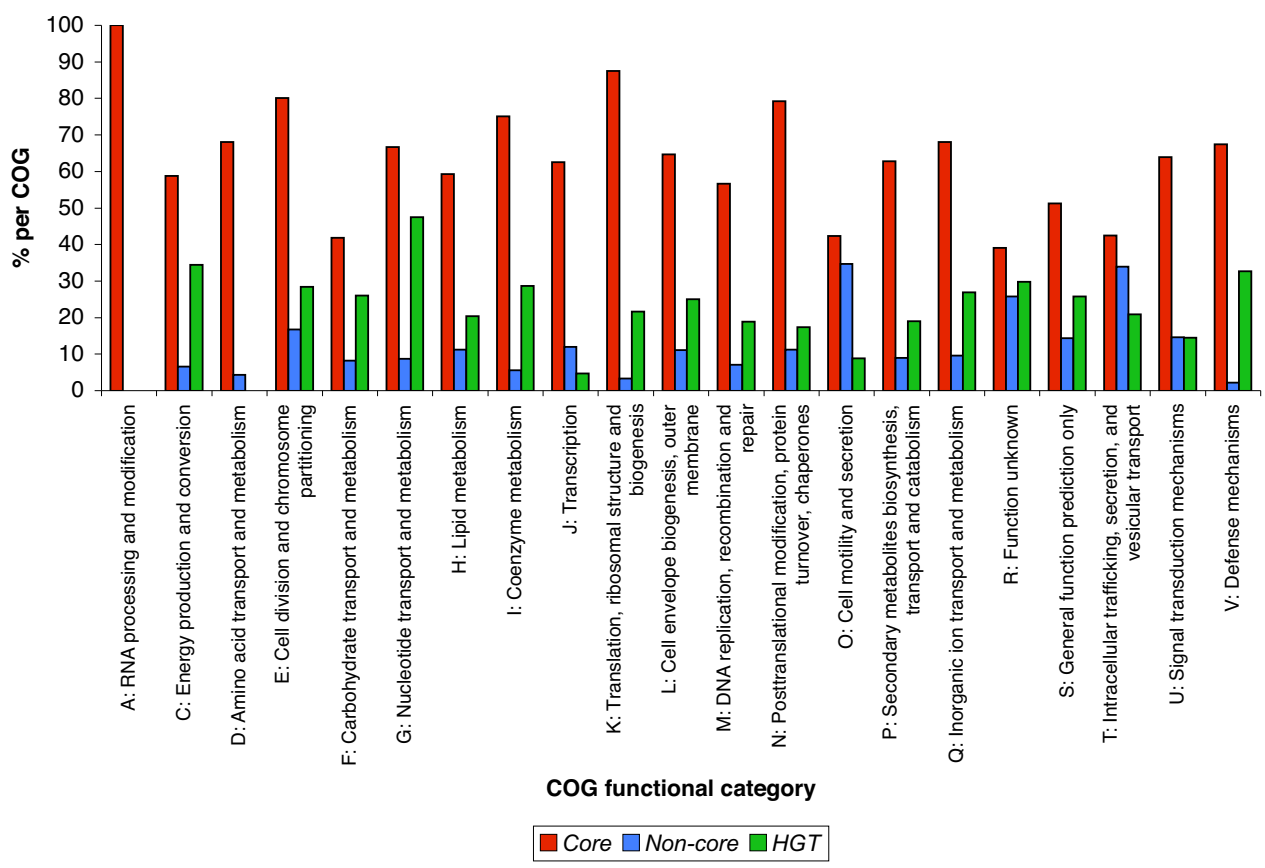

Figure 5

Distribution among the COG categories for all the E. coli genes. Counts were estimated for each evolutionary gene category, and expressed as percentages per total number of genes per COG category. The Core, Non-core and HGT gene sets differ in their distribution of COG functional categories (P-value $<0.00 \mathrm{I})$. 


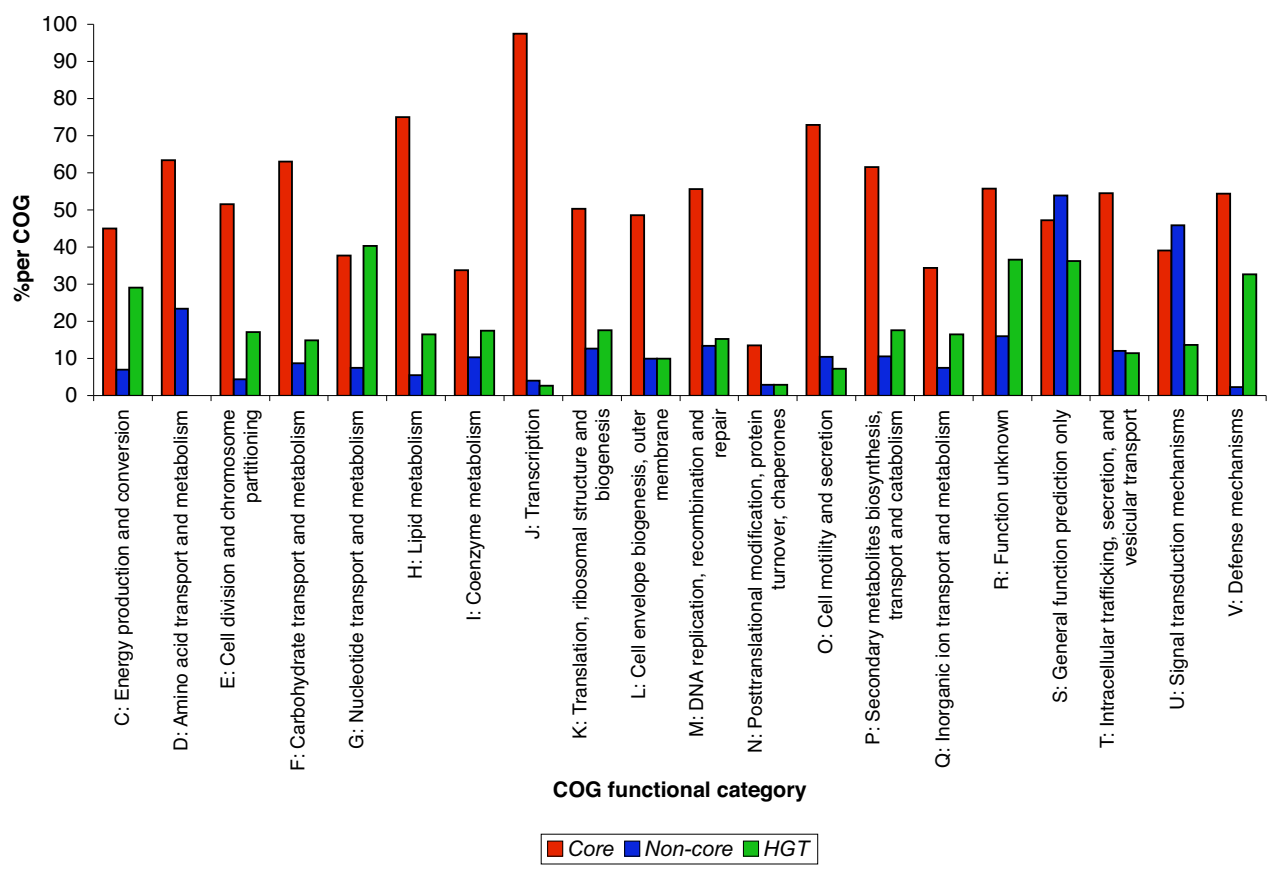

Figure 6

Distribution among the COG categories for those E. coli genes that are members of operons. Counts were estimated for each evolutionary gene category, and expressed as percentages per total number of genes per COG category. The Core, Non-core and HGT gene sets contained within operons differ in their distribution of COG functional categories $(\mathrm{P}$-value $<0.00 \mathrm{I})$.

\section{Network topology of the E. coli genes}

To investigate the mode and mechanism of integration of horizontally transferred genes into the E. coli protein-protein interaction network, we systemically investigated the network characteristics of proteins encoded by the various evolutionary categories of genes (Table 1). We found that proteins corresponding to the Core gene set represent the most highly connected protein nodes, which have an average connectivity of 11.0 interactors (Chi-squared test, pvalue $<0.05)$. In contrast, Non-core proteins and proteins encoded by HGT genes have on average lower connectivities of 4.0 and 3.0 interactors respectively. This is consistent with our hypothesis that Core genes being the most highly conserved genes have resided in the genome for much longer, and thus had more opportunities to evolve interactions. The result of the network analysis is consistent with this theory.

We also analyzed two additional network properties: betweenness centrality and clustering coefficient (Table 1). Betweenness centrality characterizes how essential a node is in maintaining communication between each pair of nodes in a network [48]. Depending on its position within the network, removal of a node can have very different effects on the connectivity, topology and flux of the network. Some nodes can be removed without any harmful effect, while others separate a connected network into disconnected sub-graphs. Betweeness centrality is a measure devised to describe the fraction of shortest paths going through a given node, with high values indicating that a node can reach many other nodes. Removal of nodes with high centrality will make it difficult to reach from one node to another, thus lengthen the path between nodes. The clustering coefficient describes the local transitivity in a network, with two nodes having a common neighbour in a network being more likely to be neighbours [49].

Table 1 shows that the HGT genes have lower betweenness centrality than the Core and Non-core genes, which suggests that they are less important in cellular communications. Interestingly the Non-core genes have higher betweeness centrality than the Core genes, the implication of which need to be further explored. On the other hand, Core genes have the highest clustering coefficients, with any two Core genes having a common neighbour being more likely to be neighbours of each other. The results in Table 1 indicate the HGT genes are the least important in maintaining the overall connectivity of the protein interaction network, in other words they are more likely to be peripheral nodes.

Our analysis of the distribution of COG functional categories of the Core, Non-core and HGT nodes within the E. coli protein interaction network reveal that the Core genes 


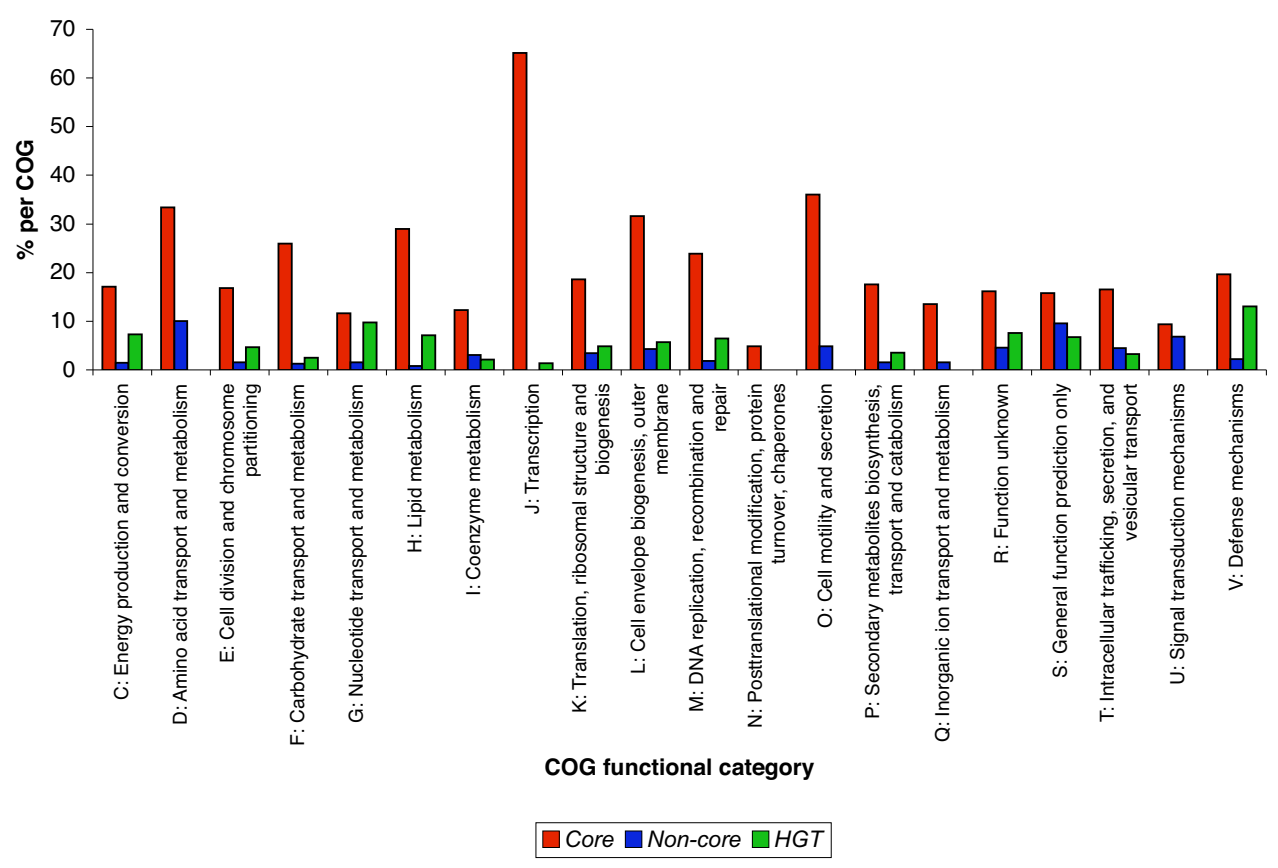

Figure 7

Distribution among the COG categories for those E. coli genes that are members of proten-protein interaction network. Counts were estimated for each evolutionary gene category, and expressed as percentages per total number of genes per COG category. The Core, Non-core and HGT gene sets contained within the protein interaction network differ in their distribution of COG functional categories ( $\mathrm{P}$-value $<0.00 \mathrm{I})$.

are the most abundant and cover all the major COG functional categories in comparison to the Non-core and HGT gene sets (Figure 7). Although, the Non-core and HGT genes show similar COG distribution profiles within the protein interaction network, differences exist in COG categories $\mathbf{C}, \mathrm{G}, \mathbf{H}$ and $\mathbf{V}$ in which the HGT genes are relatively more abundant than Non-core genes. A most notable result in this regard is the COG defense category (V) in which the HGT gene set within the E. coli protein interaction network has a significantly higher mean node degree than the Core and Non-core genes set.

Preferential Attachment of HGT proteins to Core proteins We further investigated the evolutionary profiles of the interaction partners in the network. Table 2 shows that about $74 \%$ of all the interactions are between a pair of

Table I: Protein interaction network characteristics of E. coli Core, Non-core and HGT genes

\begin{tabular}{llll}
\hline Characteristic & Core & Non-core & HGT \\
\hline Total nodes (1276) & $852(66.8 \%)$ & $178(14.0 \%)$ & $232(18.2 \%)$ \\
Ave. Node degree & 10.9 & 4.1 & 3.4 \\
$\begin{array}{l}\text { Ave. clustering coefficient } \\
\text { Ave. betweenness }\end{array}$ & 0.100 & 0.072 & 0.039 \\
centrality & $2.59 \mathrm{e}-3$ & $6.3 \mathrm{e}-4$ & $5.5 \mathrm{e}-4$ \\
\hline
\end{tabular}

Core genes, $11.2 \%$ of the interactions are between a Core gene and a Non-core gene. In other words, in total about $85 \%$ of the interactions involve at least one Core gene. Among the interactions involving HGT genes, a large percentage ( $89 \%$ ) was between a HGT genes and a Core gene, while interactions between Non-core and HGT genes only account for $1 \%$. This is surprising since the ratio between Core genes and Non-core genes is only $\sim 5: 1$, much smaller than the $9: 1$ ratio ( $89 \%: 10 \%)$ that we observed in the network. This discrepancy in ratio implies that an HGT gene have a higher propensity to establish interaction with a Core gene than with a Non-core gene. Indeed, the proportions of HGT-Core interactions are higher than expected by chance (Chi-squared test, $\mathrm{p}$-value $<0.001$ ).

Table 2: Classification of interactions based on the evolutionary profiles of interaction partners.

\begin{tabular}{ll}
\hline Category of interacting partners & Number of Interactions \\
\hline Core to Core & $3981(74.0 \%)$ \\
Non-core to Non-core & $35(0.6 \%)$ \\
HGT to HGT & $24(0.4 \%)$ \\
Core to Non-core & $606(11.2 \%)$ \\
Core to HGT & $687(12.8 \%)$ \\
Non-core to HGT & $55(1.0 \%)$ \\
Total Interactions & $5388(100 \%)$ \\
\hline
\end{tabular}


Such a model of preferential attachment has previously been proposed to explain the growth of protein interaction networks in S. cerevisiae $[20,50,51]$ and was also suggested recently for E. coli [52]; however it has remained mostly unproven since it was difficult to trace back the evolution history of protein networks. Along this line, the $H G T$ genes in E. coli offer a unique opportunity to test this theory since these genes are indeed "new genes" that were only added to the network after the HGT event 100 millions ago [5]. Our observation provided direct evidence and support for this model, which has not been reported previously.

\section{Data Availability}

Additional file 6 contains the data used and produced in this study.

\section{Conclusion}

To our knowledge, our analysis represent the first time that the HGT events are investigated in the context of protein-protein interaction and cellular networks. This is important because horizontal gene transfer in known to be prevalent in bacterial genome evolution in shaping the genome content, and they had an impact on the stability and evolution of the protein interactions and network.

From our analyses, the distinguishing characteristics which sets the HGT gene category apart from the Non-core and Core gene categories are (i) higher evolutionary substitution rates $(\mathrm{Ka} / \mathrm{Ks})$, (ii) protein interaction network statistical properties such as protein degree connectivity, average clustering coefficients and betweeness centrality, (iii) preferential attachment with regards to the number of interactions formed by HGT genes, which indicate that HGT proteins preferentially neither self-associate nor do HGT proteins associate with Non-core proteins within the E. coli protein interaction network.

Results from our study revealed a clear relationship between gene expressivity, evolutionary rate and protein connectivity for the three evolutionary classes of genes (Figure 8). The conserved Core set of genes generally display higher gene expressivity and protein connectivity than strain-specific Non-core and HGT genes. However, both gene expressivity and protein connectivity are inversely related to evolutionary rates, with the most highly conserved genes evolving the slowest. In contrast, horizontally transferred genes evolve at considerably higher evolutionary rates, and have lower gene expressivity and protein connectivity. In addition, proteins encoded by horizontally transferred genes attach preferentially to Core proteins within the E. coli protein interaction network. Consistent with this finding is the general idea that Core genes are the oldest resident genes and form the backbone of the protein interaction network to which

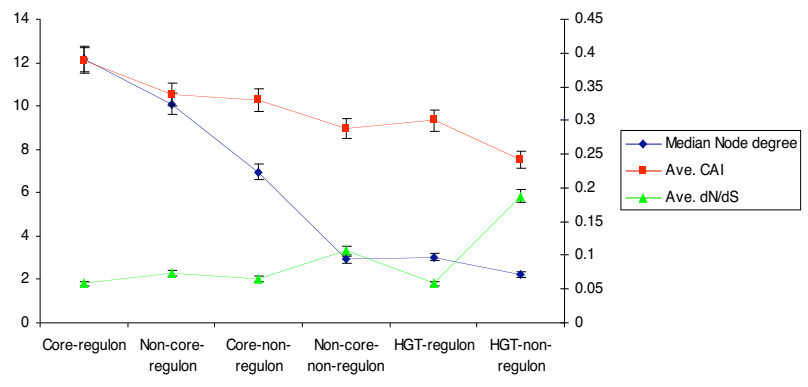

\section{Figure 8}

Summary of the relationship between protein connectivity, gene expressivity $(\mathrm{CAl})$ and evolutionary rates $(\mathrm{dN} / \mathrm{dS})$ in $E$. coli.

new proteins are attached. These results may also suggest that a proportion of the lowest connectivity proteins in bacterial protein interaction networks are those genes which are more likely to have recently been transferred and incorporated into the E. coli genome.

This is reminiscent of the so-called "Complexity Hypothesis", which was proposed to explain why the successful horizontal transfer of a gene is less probable if the connectivity of the protein it encodes is large [52], and its later modification called the 'Extended Complexity hypothesis' [53] which aims to explain why adaptive evolution is the least likely for proteins with high complexity. Although the Complexity Hypothesis and its modified version aim to describe which types of genes are more or less likely to be subjected to horizontal gene transfer, it fails to provide a mode and mechanism for subsequent integration of the horizontally transferred gene into it new recipient genome. The results from our analysis support these hypotheses with genomics and evolutionary data.

Considering the prevalence of HGT in bacteria, the relative contribution of HGT as an additional mechanism to gene duplication may become more important on network evolution. Thus, with the availability of proteomics data for more bacteria, we will most likely gain more insight on the impact of HGT on the evolution of networks.

\section{List of abbreviation used}

HGT: Horizontal Gene Transfer

PIN: Protein Interaction Network

CAI: Codon Adaption Index

BLAST: Basic Local Alignment and Search Tool 
KEGG: Kyoto Encyclopedia of Genes and Genomes http:/ Lwww.genome.jp/kegg

COG: clusters of orthologous groups http:// www.ncbi.nih.gov/COG

\section{Authors' contributions}

WD collected data, carried out the calculations, performed statistical analyses, and drafted the manuscript. WD and ZZ designed the study. ZZ participated in writing the manuscript. All authors read and approved the final manuscript.

\section{Additional material}

\section{Additional file 1}

Comparison between different HFT gene detection methods. (A) This is a 4-way comparison Venn diagram illustrating the intersection and differences between various horizontal gene transfer detection methods investigated. The comparison included a non-surrogate phylogeny and gene presence/absence based method developed by Price [15] versus three surrogate methods which included HGT-DB [27], the method published by Mrazek and Karlin [28] and a support vector machine-based method (HGT_SVM) developed by Tsirigos and Rigoutsos [29]. (B): This is a comparison of Cluster of Orthologous Group (COG) functional categories between Core, Non-core and HGT gene sets obtained using various methods of horizontal gene transfer detection. The comparison included a non-surrogate phylogeny and gene presence/absence based method developed by Price [15] versus three surrogate methods which included HGT$D B$ [27], the method published by Mrazek and Karlin [28] and a support vector machine-based method (HGT_SVM) developed by Tsirigos and Rigoutsos[29].

Click here for file

[http://www.biomedcentral.com/content/supplementary/14712148-8-23-S1.pdf]

\section{Additional file 2}

Codon usages between core, Non-core and HGT genes. This is a correspondence analysis of codon usage from $\mathrm{E}$. coli Core, Non-core, and putative HGT genes using the first two principal components.

Click here for file

[http://www.biomedcentral.com/content/supplementary/14712148-8-23-S2.pdf]

\section{Additional file 3}

Comparison between two E. coli interaction studies. This is a comparison of COG functional classes between Arifuzzaman et al. (2006) and Butland et al (2005) E. coli protein interaction networks.

Click here for file

[http://www.biomedcentral.com/content/supplementary/1471-

2148-8-23-S3.pdf]

\section{Additional file 4}

Comparison between two E. coli interaction studies. This is a comparison between Arifuzzaman et al. (2006) and Butland et al. (2005) published protein interaction data sets.

Click here for file

[http://www.biomedcentral.com/content/supplementary/14712148-8-23-S4.pdf]

\section{Additional file 5}

Statistical tests for the COG distribution. (A) Kruskal-Wallis ANOVA with Scheirer-Ray-Hare extension on the ranks of COG category counts in the Genome. (B) Kruskal-Wallis ANOVA with Scheirer-Ray-Hare extension on the ranks of COG category counts in the Operons. (C) KruskalWallis ANOVA with Scheirer-Ray-Hare extension on the ranks of COG category counts in the protein interaction network (PPI).

Click here for file

[http://www.biomedcentral.com/content/supplementary/14712148-8-23-S5.pdf]

\section{Additional file 6}

Data_2008_0117.zip. Compressed zip file containing data used in the study

Click here for file

[http://www.biomedcentral.com/content/supplementary/14712148-8-23-S6.zip]

\section{Acknowledgements}

We kindly acknowledge Morgan Price for providing the data pertaining to horizontally transferred genes; we are also grateful to the help suggestions provided by anonymous reviewers. The authors acknowledge funding support from Genome Canada through Ontario Genomic Institute.

\section{References}

I. Ochman H, Lawrence JG, Groisman EA: Lateral gene transfer and the nature of bacterial innovation. Nature 2000, 405(6784):299-304.

2. Lawrence JG, Ochman H: Reconciling the many faces of lateral gene transfer. Trends Microbiol 2002, I0(I):I-4.

3. Kurland CG, Canback B, Berg OG: Horizontal gene transfer: a critical view. Proc Natl Acad Sci USA 2003, I00(17):9658-9662.

4. Lawrence JG, Ochman H: Molecular archaeology of the Escherichia coli genome. Proc Natl Acad Sci USA 1998, 95(16):94I3-94I7.

5. Doolittle RF, Feng DF, Tsang S, Cho G, Little E: Determining divergence times of the major kingdoms of living organisms with a protein clock. Science 1996, 27 I (5248):470-477.

6. Subramanian S, Kumar S: Gene expression intensity shapes evolutionary rates of the proteins encoded by the vertebrate genome. Genetics 2004, I68(I):373-38I.

7. Drummond DA, Bloom JD, Adami C, Wilke CO, Arnold FH: Why highly expressed proteins evolve slowly. Proc Natl Acad Sci USA 2005, 102(40): 14338-14343.

8. Lawrence JG, Roth JR: Selfish operons: horizontal transfer may drive the evolution of gene clusters. Genetics 1996, I43(4): 1843-1860.

9. Lawrence JG: Selfish operons and speciation by gene transfer. Trends Microbiol 1997, 5(9):355-359.

10. Lawrence J: Selfish operons: the evolutionary impact of gene clustering in prokaryotes and eukaryotes. Curr Opin Genet Dev 1999, 9(6):642-648.

II. Omelchenko MV, Makarova KS, Wolf YI, Rogozin IB, Koonin EV: Evolution of mosaic operons by horizontal gene transfer and gene displacement in situ. Genome Biol 2003, 4(9):R55.

12. Price $M N$, Huang $\mathrm{KH}$, Arkin AP, Alm Ej: Operon formation is driven by co-regulation and not by horizontal gene transfer. Genome Res 2005, I 5(6):809-8I9.

13. Marri PR, Hao W, Golding GB: The role of laterally transferred genes in adaptive evolution. BMC Evol Biol 2007, 7(Suppl I):S8.

14. Hao W, Golding GB: The fate of laterally transferred genes: life in the fast lane to adaptation or death. Genome Res 2006, 16(5):636-643.

15. Price MN, Dehal PS, Arkin AP: Horizontal gene transfer and the evolution of transcriptional regulation in Escherichia coli. Genome Biol 2008, 9(I):R4. 
16. Itoh T, Takemoto K, Mori H, Gojobori T: Evolutionary instability of operon structures disclosed by sequence comparisons of complete microbial genomes. Mol Biol Evol 1999, 16(3):332-346.

17. Moreno-Hagelsieb G, Collado-Vides J: Operon conservation from the point of view of Escherichia coli, and inference of functional interdependence of gene products from genome context. In Silico Biol 2002, 2(2):87-95.

18. Light $S$, Kraulis P, Elofsson A: Preferential attachment in the evolution of metabolic networks. Bmc Genomics 2005, 6:

19. Pal C, Papp B, Lercher MJ: Adaptive evolution of bacterial metabolic networks by horizontal gene transfer. Nat Genet 2005 37(12): 1372-1375.

20. Barabasi AL, Albert R: Emergence of scaling in random networks. Science 1999, 286(5439):509-5I 2 .

21. Ragan MA, Charlebois RL: Distributional profiles of homologous open reading frames among bacterial phyla: implications for vertical and lateral transmission. Int J Syst Evol Microbiol 2002, 52(Pt 3):777-787.

22. Daubin V, Ochman $\mathrm{H}$ : Quartet mapping and the extent of lateral transfer in bacterial genomes. Mol Biol Evol 2004 2I(I):86-89

23. microbesonline.org [http://www.microbesonline.org]

24. Edgar RC: MUSCLE: multiple sequence alignment with high accuracy and high throughput. Nucleic Acids Res 2004, 32(5): $1792-1797$.

25. Howe K, Bateman A, Durbin R: QuickTree: building huge Neighbour-Joining trees of protein sequences. Bioinformatics 2002, I 8(I I): I546-I547.

26. Schmidt HA, Strimmer K, Vingron M, von Haeseler A: TREE-PUZ ZLE: maximum likelihood phylogenetic analysis using quartets and parallel computing. Bioinformatics 2002, I 8(3):502-504.

27. Garcia-Vallve S, Guzman E, Montero MA, Romeu A: HGT-DB: a database of putative horizontally transferred genes in prokaryotic complete genomes. Nucleic Acids Res 2003 , $31(1): 187-189$.

28. Mrazek J, Karlin S: Detecting alien genes in bacterial genomes. Ann N Y Acad Sci 1999, 870:3।4-329.

29. Tsirigos A, Rigoutsos I: A sensitive, support-vector-machine method for the detection of horizontal gene transfers in viral, archaeal and bacterial genomes. Nucleic Acids Res 2005 33(I 2):3699-3707.

30. Lawrence JG, Ochman H: Amelioration of bacterial genomes: rates of change and exchange. J Mol Evol 1997, 44(4):383-397.

31. Koski LB, Morton RA, Golding GB: Codon bias and base composition are poor indicators of horizontally transferred genes. Mol Biol Evol 200I, I8(3):404-4I2.

32. Huerta $A M$, Salgado $H$, Thieffry $D$, Collado-Vides J: RegulonDB: a database on transcriptional regulation in Escherichia coli. Nucleic Acids Res 1998, 26( I):55-59.

33. Salgado H, Gama-Castro S, Peralta-Gil M, Diaz-Peredo E, SanchezSolano F, Santos-Zavaleta A, Martinez-Flores I, Jimenez-Jacinto V, Bonavides-Martinez C, Segura-Salazar J, Martinez-Antonio A, Collado-Vides J: RegulonDB (version 5.0): Escherichia coli K-I2 transcriptional regulatory network, operon organization, and growth conditions. Nucleic Acids Res 2006:D394-397.

34. Barrett T, Suzek TO, Troup DB, Wilhite SE, Ngau WC, Ledoux P, Rudnev D, Lash AE, Fujibuchi W, Edgar R: NCBI GEO: mining millions of expression profiles - database and tools. Nucleic Acids Res 2005:D562-566.

35. Butland G, Peregrin-Alvarez JM, Li J, Yang W, Yang X, Canadien V, Starostine A, Richards D, Beattie B, Krogan N, Davey M, Parkinson J, Greenblatt J, Emili A: Interaction network containing conserved and essential protein complexes in Escherichia coli. Nature 2005, 433(7025):53I-537.

36. Arifuzzaman M, Maeda M, Itoh A, Nishikata K, Takita C, Saito R, Ara T, Nakahigashi K, Huang HC, Hirai A, Tsuzuki K, Nakamura S, AltafUl-Amin M, Oshima T, Baba T, Yamamoto N, Kawamura T, lokaNakamichi T, Kitagawa M, Tomita M, Kanaya S, Wada C, Mori H: Large-scale identification of protein-protein interaction of Escherichia coli K-I2. Genome Res 2006, 16(5):686-69I.

37. Wall DP, Fraser HB, Hirsh AE: Detecting putative orthologs. Bioinformatics 2003, 19(13):|7|0-|7||1.

38. Thompson JD, Higgins DG, Gibson T]: CLUSTAL W: improving the sensitivity of progressive multiple sequence alignment through sequence weighting, position-specific gap penalties and weight matrix choice. Nucleic Acids Res 1994, 22(22):4673-4680

39. Saitou N, Nei M: The neighbor-joining method: a new method for reconstructing phylogenetic trees. Mol Biol Evol 1987, 4(4):406-425

40. Yang Z: PAML: a program package for phylogenetic analysis by maximum likelihood. Comput Appl Biosci 1997, 13(5):555-556.

41. NetworkX package [https://networkx.lanl.gov]

42. Batagelj v, Mrvar A: Pajek - Program for large network analysis. Connections 1998, 2 I (2):47-57.

43. Gouy $M$, Gautier $C$ : Codon usage in bacteria: correlation with gene expressivity. Nucleic Acids Res 1982, 10(22):7055-7074.

44. Williams EJ, Hurst LD: The proteins of linked genes evolve at similar rates. Nature 2000, 407(6806):900-903.

45. Hurst LD, Williams EJ, Pal C: Natural selection promotes the conservation of linkage of co-expressed genes. Trends Genet 2002, I 8( I 2):604-606.

46. Burland V, Shao Y, Perna NT, Plunkett G, Sofia HJ, Blattner FR: The complete DNA sequence and analysis of the large virulence plasmid of Escherichia coli O157:H7. Nucleic Acids Res 1998, 26( I 8):4196-4204.

47. Jensen RA: Enzyme recruitment in evolution of new function. Annu Rev Microbiol 1976, 30:409-425.

48. Freeman L: A set of measures of centrality based on betweenness. Sociometry 1977, 40:35-4I.

49. Watts DJ, Strogatz SH: Collective dynamics of 'small-world' networks. Nature 1998, 393(6684):440-442.

50. Eisenberg E, Levanon EY: Preferential attachment in the protein network evolution. Phys Rev Lett 2003, 9 I (13): I3870I.

51. Kunin V, Pereira-Leal JB, Ouzounis CA: Functional evolution of the yeast protein interaction network. Mol Biol Evol 2004, 2I(7): II7|-II76.

52. Jain R, Rivera MC, Lake JA: Horizontal gene transfer among genomes: the complexity hypothesis. Proc Natl Acad Sci USA 1999, 96(7):380I-3806.

53. Aris-Brosou S: Determinants of adaptive evolution at the molecular level: the extended complexity hypothesis. Mol Biol Evol 2005, 22(2):200-209.
Publish with BioMed Central and every scientist can read your work free of charge

"BioMed Central will be the most significant development for disseminating the results of biomedical research in our lifetime. "

Sir Paul Nurse, Cancer Research UK

Your research papers will be:

- available free of charge to the entire biomedical community

- peer reviewed and published immediately upon acceptance

- cited in PubMed and archived on PubMed Central

- yours - you keep the copyright
BioMedcentral 